AVALIAÇÃO FÍSICO-QUÍMICA E MICROBIOLÓGICA DA DIPIRONA SÓDICA E SUA RELAÇÃO COM O PERFIL DE ARMAZENAMENTO DOMICILIAR

\title{
PHYSICAL-CHEMICAL AND MICROBIOLOGICAL EVALUATION OF DIPYRONE AND ITS RELATIONSHIP WITH STORAGE HOUSEHOLD'S PROFILE
}

\author{
SCHINDLER ${ }^{1}$, F. R.; YAMANAKA², E. H. U.; COMARELLA ${ }^{3}$, L.; JUNIOR ${ }^{4}$, D. A.
}

1 - Acadêmica do Centro Universitário Campos de Andrade.

2 - Professora Mestre do Curso de Farmácia do Centro Universitário Campos de Andrade.

3 - Professora Mestre do Curso de Farmácia do Centro Universitário Campos de Andrade.

4 - Professor Mestre do Curso de Farmácia do Centro Universitário Campos de Andrade.

Email: cientifico@laborclin.com.br

\section{RESUMO:}

A dipirona é um dos analgésicos mais populares no Brasil, estando presente na maioria das "farmácias caseiras". Essa prática de estocagem domiciliar, geralmente encontrase de forma inadequada, o que pode levar a alterações físico-químicas e microbiológicas do produto farmacêutico. $O$ objetivo deste trabalho foi verificar alterações na estabilidade de diferentes formas farmacêuticas de dipirona sódica, bem como comparar as características de armazenamento de medicamentos em residências. Para tal, foram simuladas em diferentes condições de armazenamento amostras de comprimidos, solução oral e xarope, durante quatro meses, sendo posteriormente analisadas conforme critérios estabelecidos pela Farmacopeia Brasileira $5^{a}$ edição. Para apresentações da dipirona solução oral e xarope, foram realizados testes de $\mathrm{pH}$ e segurança biológica (contagem de micro-organismo mesófilos e bolores/leveduras); para os comprimidos, foram realizados os testes de friabilidade, desintegração e doseamento. Os resultados obtidos das análises apresentaram determinações de pH entre 6,11 e 6,96 (dentro dos padrões); não observou-se crescimento de micro-organismos aeróbios e o desenvolvimento de fungos permaneceu $<10^{1} \mathrm{UFC} / \mathrm{g}$; todas as amostras foram desintegradas em menos de 10 minutos; para o teste de friabilidade detectaram-se perdas não superiores a $0,60 \%$ e para o teor do fármaco as perdas se mantiveram inferiores a $10 \%$ do valor nominal. Assim, pode-se concluir que, durante o período da simulação, nenhuma das amostras apresentou variações de acordo com padrões farmacopeicos. Porém, se a exposição aos fatores ambientais continuar associada a um tempo mais prolongado de armazenamento e à falta de higiene na manipulação, os medicamentos estão sujeitos a sofrer alterações físico-químicas e microbianas.

Palavras-chave: armazenamento domiciliar; dipirona sódica; estabilidade.

\section{ABSTRACT:}

The dipyrone is one of the most popular analgesics in Brazil, being present in most "home pharmacy". This practice of home storage it is usually in an inappropriately way, which can lead to physical and chemical and microbiological changes of the pharmaceutical product. The objective of this study was to assess changes in the stability of different pharmaceutical forms of sodium dipyrone as well as to compare the drug home storage's characteristics. To this end, they were simulated in different storage 
conditions tablets, oral solution and syrup sample, for four months, and subsequently analyzed according to criteria established by the Brazilian Pharmacopoeia 5th edition. For dipyrone oral and syrup solution presentations, $\mathrm{pH}$ tests and biological safety were performed (mesophilic microorganism count and molds/yeasts); for tablets, the friability tests were performed, disintegration and dosing. The results of the analyzes showed $\mathrm{pH}$ between 6.11 and 6.96 determinations (within the standards); not observed growth of aerobic micro-organisms and the development of fungi remained $<10^{1} \mathrm{CFU} / \mathrm{g}$; all samples were disintegrated in less than 10 minutes; for the test friability were detected loss of not more than $0.60 \%$ and the drug content of the losses remained below $10 \%$ of nominal. Thus, it can be concluded that during the simulation period, none of the samples showed variations according to pharmacopoeia standards. However, if exposure to environmental factors remains associated with a longer storage time and lack of hygiene in handling, the drugs are subject to suffer physical, chemical and microbial changes.

Keywords: home storage; dipyrone; stability.

\section{INTRODUÇÃO}

Os medicamentos conquistaram um lugar de evidência na terapêutica moderna, apontando uma grande evolução no desenvolvimento da saúde pública (NASCIMENTO, 2005). Porém, esses produtos estão se acumulando nas residências, devido ao seu rotineiro uso, aliado a facilidade de aquisição e o estímulo da mídia, fazendo com que a população se considere isenta dos riscos inerentes a conservação (FERNANDES e PETROVICK, 2004).

Ao serem dispensados para o consumidor, os medicamentos precisam de cuidados com o seu armazenamento, já que este é um fator importante na preservação de seus componentes, visando à garantia de sua ação farmacológica (BRASIL, 2009). O acondicionamento inadequado pode levar a alterações físicas, químicas e microbiológicas, resultando em atividade reduzida ou formação de produtos tóxicos (CARVALHO etal., 2009).

Produtos farmacêuticos têm sua estabilidade dependente de fatores ambientais, tais como temperatura, luz e umidade, além de outros relacionados à própria substância, como propriedades físicas e químicas de ativos e excipientes, sua composição e forma farmacêutica, materiais de embalagem e processo de fabricação (BRASIL, 2005).

A Agência Nacional de Vigilância Sanitária (ANVISA), para assegurar a qualidade dos medicamentos, exige no processo de fabricação a garantia das características destes produtos, sendo fundamental que, após a dispensação ao consumidor, os mesmos também sejam mantidos em condições ideais nos estoques domiciliares (FERREIRAet al., 2014).

Um dos analgésicos mais vendidos no Brasil, a dipirona, também chamada de metamizol, é considerada um dos medicamentos mais populares, devido sua 
acessibilidade e baixo custo. Em formulações para uso oral é amplamente utilizada como equivalente de ácido acetilsalicílico e paracetamol (KOROLKOVAS e BURCKHALTER, 1988; FERREIRA, 2010).

Apesar de ser um anti-inflamatório não esteroidal (AINE) fraco, é um potente analgésico e antipirético, indicado para patologias como cefaleias, neuralgias, dores reumáticas, cólica renal e dor pós-operatória (KNAPPMANN e MELO, 2010).

Porém, o uso desse analgésico é controverso em alguns países, como Estados Unidos e outros da Europa, onde já foi banido em consequência à ocorrência de reações alérgicas graves como: edema de glote, anafilaxia e discrasias sanguíneas (KÖHLER et al., 2009).

No Brasil, em um encontro sobre a segurança da dipirona (patrocinado pela ANVISA), chegou-se à conclusão que, quando comparada a outros analgésicos/antipiréticos presentes no mercado, apresenta a garantia e a eficácia necessária para continuar a ser comercializada no país, como um medicamento isento de prescrição (AGÊNCIANACIONAL DE VIGILÂNCIASANITÁRIA, 2001).

Tendo em vista que grande parte da população apresenta uma "farmácia caseira", onde comumente os medicamentos encontram-se em condições não apropriadas, este trabalho tem como objetivo verificar alterações na estabilidade de diferentes formas farmacêuticas de dipirona sódica, bem como comparar as condições e características de armazenamento de medicamentos em residências.

\section{REFERENCIAL TEÓRICO}

A prática de estocagem de medicamentos em residências ocorre, principalmente, por métodos preventivos da população contra o aparecimento de doenças e sintomas (MARGONATO, THOMSON e PAOLIELLO, 2008).

Em um trabalho realizado por Figueiredo e colaboradores (2008), onde foram pesquisadas "farmácias caseiras" no bairro de Figueirinha, em Xangri-lá-RS, a cozinha foi o ambiente mais escolhido (58\%), geralmente dentro de algum armário (36\%) ou sobre este (14\%). Ainda, atribuído por Tótoli e autores (2011), ao fato desse ambiente proporcionar maior facilidade ao acesso e a administração. Embora, seja um cômodo não apropriado, pois pode provocar o aceleramento da degradação de fármacos, devido ao aumento da temperatura (MILANEZ et al., 2013).

Conforme Tourinho et al. (2008), que avaliaram o estoque domiciliar de medicamentos em uma cidade em São Paulo, os principais cômodos foram dormitório $(47,5 \%)$, cozinha $(29,9 \%)$ e banheiro $(14,6 \%)$. Os autores também indicam que as variações quanto ao local mais utilizado no armazenamento domiciliar de medicamentos têm influência cultural e regional.

Outros locais, como em cima da geladeira e próximo ao fogão e/ou forno micro-ondas, 
foram citados pelos moradores em outros estudos. Dados encontrados na pesquisa feita por Ferreira e colaboradores (2005), em Porto Alegre-RS, mostraram que a maior porção dos medicamentos encontrados na cozinha estava principalmente nos armários e sobre estes eletrodomésticos. Porém, também de forma inadequada, uma vez que o aquecimento do motor e a radiação podem causar alterações aos produtos.

Com uma menor proporção, mas ainda utilizado, o banheiro é um ambiente exposto à umidade e micro-organismos, o que pode levar à degradação da substância ativa e alterações na molécula (SERAFIM et al., 2007).

Contudo, a falta de cuidados com a farmácia domiciliar pode alterar a eficiência e comprometer a segurança no uso dos medicamentos. Segundo Figueiredo e pesquisadores (2011), o local destinado ao armazenamento deve ser em ambiente seco, ao abrigo da luz e do calor, evitando assim a possíveis alterações do produto.

Mastroianni e colaboradores (2011) observaram que 90,9\% dos entrevistados guardam o medicamento apenas na embalagem primária. Isso, de acordo com os autores, torna-se um risco de segurança, pois o produto não recebe proteção e a sua rastreabilidade fica comprometida.

Além disso, a observação do aspecto/aparência do medicamento é de extrema importância, para garantir que o mesmo não esteja em degradação visível. Ferreira e colaboradores (2005) encontraram $28,61 \%$ das pessoas que administram os medicamentos sem observar essas alterações. Por sua vez, Bueno, Weber e Oliveira (2009) constataram que 75,39\% dos entrevistados também não o observam.

Através das características organolépticas dos medicamentos, os sentidos sensoriais permitem com razoável facilidade a identificação da perda da estabilidade física. No entanto, é importante associar que, quando a integridade visualmente pode ser percebida alterada, as estabilidades química e microbiológica já estão possivelmente comprometidas, ocasionando prejuízos consideráveis para a ação terapêutica (REIS et al., 2003).

\section{MATERIAIS E MÉTODOS}

\subsection{Metodologia}

O presente estudo trata-se de uma pesquisa quantitativa, descritiva e explicativa sobre a avaliação físico-química e microbiológica da dipirona sódica, comparada às diferentes condições de armazenamento domiciliar.

Buscou-se, através das análises, a identificação das alterações e contaminantes, referente às formas farmacêuticas: comprimidos, solução oral e xarope, nas apresentações de blisters, frascos conta-gotas e frascos com copomedida. 
Os resultados foram registrados no programa Excel 2010. Passaram por interpretação numérica, com tabelamento em percentual estatístico.

\subsection{Seleção da amostra de medicamentos}

A amostragem de medicamentos abrangeu aproximadamente 400 comprimidos, 15 frascos de solução conta-gotas e 5 frascos de xarope com copomedida, preconizado para cada forma farmacêutica a utilização sempre do mesmo laboratório.

Todas as amostras se encontravam dentro do prazo de validade e foram retiradas de suas respectivas embalagens secundárias.

No decorrer de quatro meses (Dezembro 2014 a Março 2015) foram simuladas situações de armazenamento de três tipos de apresentação de dipirona sódica (3 amostras diferentes) - blisters de comprimidos (500mg), solução oral $(500 \mathrm{mg} / \mathrm{mL})$ e xarope $(50 \mathrm{mg} / \mathrm{mL})$ - em cinco condições, descritas no Quadro 1, sendo escolhidos os locais frequentemente citados em estudos (SERAFIM et al., 2007; FERREIRA et al., 2014).

QUADRO 1. Casos de simulação da amostragem.

\begin{tabular}{|c|c|}
\hline LOCAIS & CONDIÇÕES \\
\hline Quarto & Sobre bancada, próxima a janela \\
\hline Sala & Dentro do armário \\
\hline Banheiro & Em cima da pia \\
\hline Cozinha(a) & Próximo ao fogão/micro-ondas \\
\hline Cozinha(b) & Em cima da geladeira \\
\hline
\end{tabular}

FONTE: Aautora, 2015.

\subsection{Avaliação da estabilidade da dipirona sódica}

As análises físico-químicas e microbiológicas foram realizadas nos laboratórios do Centro Universitário Campos de Andrade, seguindo a metodologia 
descrita na Farmacopeia Brasileira $5^{\mathrm{a}}$ edição (2010b), para os três tipos de amostras.

o caso das apresentações da dipirona solução oral, foram aplicados testes de $\mathrm{pH}$ e segurança biológica (contagem de micro-organismo mesófilos e bolores/leveduras). Para os comprimidos, foram realizados os testes de friabilidade, desintegração e doseamento (FARMACOPEIA, 2010a).

\subsection{Instrumentação e materiais}

As pesagens foram efetuadas utilizando a balança de precisão com capela Marte ${ }^{\circledR}$. Para a realização dos testes de estabilidade foram empregados os equipamentos: pHmetro Noxtron ${ }^{\circledR}$ Mod. nox68; desintegrador Nova Ética ${ }^{\circledR}$ Mod. $301 \mathrm{AC}$ e friabilômetro Nova Ética ${ }^{\circledR}$ Mod. 300.

O controle microbiológico abrangeu o uso de fluxo laminar; frascos contendo o caldo diluente TSB (crescimento microbiano) e placas de Petri contendo os meios de cultura Sabouraud-dextrose (fungos) e TSA (micro-organismos aeróbios) da Laborclin; alça de Drigalski e estufa de cultura bacteriológica Odontobrás ${ }^{\circledR}$ Mod. ECB 1.2 Digital.

\subsection{Testes e procedimentos}

\subsection{1 pH}

Após a aferição do pHmetro, com as soluções tampão pH 4,00 e pH 7,00, lavouse o eletrodo com água destilada e com várias porções da amostra. Procedeu a leitura das alíquotas de solução oral da dipirona sódica. Sendo o teste realizado em triplicata. O limite estabelecido pela Farmacopeia Brasileira $5^{\text {a }}$ edição, é de 5,5 a 7,0.

\subsubsection{Controle de micro-organismos mesófilos}

Para a realização do método de semeadura em superfície, previamente, foi preparado as amostras utilizando a diluição 1:10, com Caldo TSB. Foi inoculado 0,1 mL de cada diluição, depositando-o no centro de cada placa contendo ágar TSA e ágar Sabouraud-dextrose. Foram utilizadas duas placas para cada meio e diluição.

Após, foi incubado as placas, sem invertê-las, as quais continham ágar TSA a $32,5 \pm 2,5^{\circ} \mathrm{C}$ durante 5 dias e as placas com ágar Sabouraud-dextrose a $22,5 \pm 2,5^{\circ} \mathrm{C}$ durante 7 dias, para determinação do número de micro-organismos aeróbicos totais e bolores/leveduras, respectivamente.

Segundo os limites microbianos para produtos não estéreis, a contagem total de bactérias aeróbias não deve exceder $10^{2} \mathrm{UFC} / \mathrm{g}$ e a contagem total de fungos deve ser inferior a $10^{1}$ UFC/g (FARMACOPEIA, 2010a). 


\subsubsection{Friabilidade}

Foram pesados 20 comprimidos de dipirona sódica 500 mg e introduzidos em um friabilômetro, ajustado à velocidade para 25 rotações por minuto e o tempo de teste para 4 minutos. Decorrido o prazo, foi removido qualquer resíduo de pó da superfície dos comprimidos e pesado novamente. De acordo com as especificações farmacopeicas, são aceitáveis perdas de até $1,5 \%$.

\subsubsection{Tempo de Desintegração}

Foi colocado um comprimido em cada um dos seis tubos da cesta do desintegrador, adicionando a eles um disco. Sendo utilizado como líquido de imersão a água mantida a $37 \pm 1^{\circ} \mathrm{C}$, acionando assim o aparelho. Ao final do intervalo de 30 minutos, é cessado o movimento da cesta e observado o material em cada um dostubos. Os quais, segundo a Farmacopeia Brasileira (2010), devem estar completamente desintegrados.

\subsubsection{Doseamento}

Para a realização do teste de iodometria, foram pulverizados comprimidos. Destes, foi pesado a quantidade de pó equivalente a 0,35 g de dipirona e transferido para erlemeyer. Adicionado $25 \mathrm{~mL}$ de água, $5 \mathrm{~mL}$ de ácido acético glacial e agitado até dispersão. Então, foi titulado com iodo 0,05 M SV, utilizando amido SI como indicador, em temperatura abaixo de $15^{\circ} \mathrm{C}$. Sendo que cada $\mathrm{mL}$ gasta corresponde a $17,570 \mathrm{mg}$ de dipirona. Os limites farmacopeicos são de $\pm 10 \%$ do valor nominal.

\section{RESULTADOS}

Os testes de estabilidade realizados com a solução oral e xarope de dipirona sódica foram determinação de pH e segurança microbiológica. As análises foram avaliadas após 4 meses de armazenamento dos medicamentos e em triplicata para pH e duplicada para pesquisa microbiológica.

$\mathrm{O}$ pH inicial variou entre 6,61-6,98 (solução) e 6,38-6,54 (xarope) antes da simulação. Os resultados, após o período de armazenamento em diferentes locais, estão expostos na Tabela 1. 
TABELA 1. Resultados da determinação do $\mathrm{pH}$ para diferentes locais de armazenamento de dipirona solução e xarope.

\begin{tabular}{ccc}
\hline Amostra & Solução oral & Xarope \\
\hline Sala & 6,96 & 6,53 \\
Quarto & 6,81 & 6,48 \\
Banheiro & 6,77 & 6,42 \\
Cozinha(a) & 6,59 & 6,11 \\
Cozinha(b) & 6,65 & 6,34 \\
\hline
\end{tabular}

Pode-se perceber que, posteriormente a simulação, o pH apresentou variações dentro do estabelecido pela Farmacopeia Brasileira $5^{a}$ edição.

$\mathrm{Na}$ análise microbiológica das formas farmacêuticas líquidas, não se observou crescimento de micro-organismos aeróbios e o desenvolvimento de bolores/leveduras permaneceu $<10^{1} \mathrm{UFC} / \mathrm{g}$, em acordo com as especificações farmacopeicas (FARMACOPEIAa, 2010).

Para os comprimidos, foram analisados desintegração, friabilidade e doseamento do fármaco (conforme descrito em materiais e métodos). Apenas o ensaio de doseamento foi realizado em triplicata.

Com relação ao teste de desintegração (parâmetro farmacopeico $\leq 30 \mathrm{~min}$ ), todas as amostram foram desintegradas com menos de 10 minutos, cumprindo as especificações.

Para a friabilidade, detectaram-se perdas não superiores a $0,60 \%$, estando em acordo com os limites aceitáveis (perdas de no máximo de 1,5\%).

As porcentagens de perdas no teste de friabilidade, para comprimidos armazenados em diferentes locais, estão descritos na Tabela 2.

TABELA 2. Resultados do teste de friabilidade para diferentes locais de armazenamento de dipirona comprimido.

\begin{tabular}{cc}
\hline Amostra & Perdas (\%) \\
\hline Sala & 0,17 \\
Quarto & 0,18 \\
Banheiro & 0,35 \\
Cozinha(a) & 0,53 \\
Cozinha(b) & 0,39 \\
\hline
\end{tabular}


Para os testes de doseamento, a Farmacopeia especifica que as perdas podem ser até $\pm 10 \%$ do valor nominal. Os teores de dipirona encontrados nas amostras analisadas (Tabela 3) demonstraram a quantidade do fármaco presente e o percentual de perdas após o ensaio.

TABELA 3. Resultados do doseamento do fármaco para diferentes locais de armazenamento de dipirona comprimido.

\begin{tabular}{cccc}
\hline Amostra & $\begin{array}{c}\text { Doseamento inicial } \\
(\mathbf{m g})\end{array}$ & $\begin{array}{c}\text { Doseamento após } \\
\text { teste }(\mathbf{m g})\end{array}$ & Perdas (\%) \\
\hline Sala & 498,988 & 488,446 & 2,11 \\
Quarto & 500,745 & 481,418 & 3,86 \\
Banheiro & 498,988 & 470,876 & 5,64 \\
Cozinha(a) & 497,231 & 467,362 & 6,01 \\
Cozinha(b) & 500,745 & 472,633 & 5,62 \\
\hline
\end{tabular}

Conforme apresentado, não houve perdas superiores ao estabelecido, indicando que os comprimidos de dipirona mantêm sua estabilidade por pelo menos 4 meses, quando armazenados nos locais indicados.

\section{DISCUSSÃO}

A estabilidade dos medicamentos é caracterizada pela permanência das mesmas características e condições físico-químicas e microbiológicas da sua fabricação, mantidas dentro das especificações, durante todo o período de estocagem e uso (TABORIANSKI, 2003; STULZER e SILVA, 2006).

Nesse estudo, todas as amostras líquidas de dipirona analisadas permaneceram dentro do limite de pH estabelecido pela Farmacopeia, após 4 meses de armazenamento, em diferentes locais de uma residência. Como ensaio de qualidade de medicamentos, a determinação do pH é essencial, estando relacionada a fatores como biocompatibilidade do princípio ativo e estabilidade química do fármaco (GIL, 2007).

Semelhante ao estudo de Knappmann e Melo (2010), que analisaram marcas de dipirona comercializadas em uma drogaria de Cascavel-PR, todas as amostras se encontraram dentro do limite farmacopeico, com pH médio variando entre 5,8 e 6,8.

Porém, na pesquisa de Ferreira e colaboradores (2014), que avaliaram a estabilidade de dipirona em gotas armazenadas em residências de São Luiz de Montes Belos-GO, 
das 53 amostras analisadas, 9 foram reprovadas, por apresentarem pH inferior a 5,5. Entretanto, o estudo não considerou o período de armazenamento nas residências, apenas que as amostras estivessem dentro do prazo de validade.

Com relação às análises microbiológicas, não houve desenvolvimento microbiano em nenhuma das amostras. Acontagem de micro-organismos mesófilos foi aplicada para determinar se o medicamento satisfaz às exigências farmacopeicas, uma vez que, através dela, é possível definir o número total de bactérias e fungos em produtos não-estéreis (FARMACOPEIA, 2010a). O resultado negativo de crescimento torna-se um indicativo de Boas Práticas de Fabricação por parte das indústrias farmacêuticas e pela manipulação adequada das amostras durante a simulação.

No estudo realizado por Serafim e colaboradores (2007), que avaliaram a qualidade dos medicamentos contendo dipirona encontrados em residências de Araraquara-SP, 26,2\% das amostras analisadas apresentaram contaminação microbiológica, detectando-se Staphylococcus aureus, Escherichia coli e Salmonella sp. (importantes patógenos), além de grande número de bolores e leveduras. Neste caso também não foi determinado o período de estocagem, apenas a validade vigente.

O estudo acima indica que, apesar dos medicamentos apresentarem conservantes em sua formulação, houve desenvolvimento microbiano, o que poderia ser considerado um indicativo da má conservação e manipulação dos medicamentos em residências. Formas farmacêuticas líquidas estão mais sujeitas a proliferação de micro-organismos e, por isso, os cuidados de armazenamento devem ser redobrados.

Para verificação da qualidade de comprimidos foram utilizados os testes de desintegração, friabilidade e doseamento.

A desintegração de comprimidos, segundo Peixoto e autores (2005), afeta diretamente a absorção, a biodisponibilidade e a ação terapêutica do fármaco. O que apresentou-se favorável na análise, onde todos os comprimidos foram totalmente desintegrados.

Os resultados para friabilidade permaneceram entre 0,18 a $0,53 \%$, dentro do limite estabelecido, não ocorrendo perdas significativas. Portanto, as amostras foram aprovadas no teste. Esse ensaio apresenta a relevância de verificar a resistência dos comprimidos à perda de peso, decorrente da abrasão, choques mecânicos que podem ser submetidos em atividades do cotidiano, envolvendo o armazenamento e o manuseio pelo paciente. A alta friabilidade pode ocasionar a perda do princípio ativo, levando ao comprometimento da eficácia terapêutica e a presença de quebras e rachaduras pode provocar uma inaceitabilidade pelo paciente, devido ao mau aspecto do comprimido (PEIXOTO et al., 2005).

O doseamento de fármacos indica a quantidade de princípio ativo presente na formulação (FERREIRA et al., 2014). Os teores de dipirona encontrados nas amostras 
analisadas demonstraram perdas, porém todas encontraram-se de acordo com os padrões farmacopeicos.

Porém, os resultados obtidos por Serafim e pesquisadores (2007) revelaram a instabilidade da dipirona solução oral dentro do prazo de validade, atingindo até 42,6\% de perdas no teor dos fármacos.

Estudos realizados com outros medicamentos também demonstraram perdas de ativo. O ensaio realizado por Santos, Aragão e Furlan (2009) sobre a estabilidade da solução oral de paracetamol a partir de 60 dias de armazenamento, demonstrou que os teores deste fármaco sofreram redução abaixo do limite mínimo especificado (90\%), tanto para as situações de amostras protegidas da luz solar quanto para as expostas, ambas em temperatura ambiente.

Ainda, os resultados expostos por Stulzer e Silva (2006), que estudaram a estabilidade de comprimidos contendo captopril, no período de três meses, apontaram que o fármaco sofreu uma diminuição do seu teor quando submetido às diferentes condições de temperatura (12,65\%), umidade (13,23\%) e luz ultravioleta (42\%).

Isso indica que qualquer medicamento e forma farmacêutica podem ser afetados por condições inadequadas de armazenamento domiciliar.

O maior problema com as "farmácias caseiras" provém da facilidade de acesso aos medicamentos com a ausência de informações necessárias, que ainda, dependendo das condições de armazenamento, podem causar a contaminação e/ou degradação de seus componentes (MASTROIANNI et al., 2011).

Verificou-se entre os estudos um alto índice de falta de informação dos moradores, o que é preocupante tendo em vista que uma grande parcela da população está se expondo a um risco desnecessário e que pode ser facilmente evitado. Dentre várias funções relacionadas à prescrição e dispensação, o profissional farmacêutico também tem o dever de fornecer informações ao paciente sobre o correto armazenamento domiciliar de medicamentos (WEST, HERBERT e KNOWLTON, 2014).

\section{CONCLUSÃO}

Através dos resultados encontrados nas análises de estabilidade, pode-se concluir que durante o período de quatro meses da simulação, nenhuma das formas farmacêuticas de dipirona sódica apresentou variações de padrões estabelecidos pela Farmacopeia. Mas isso não impede que, se continuar a exposição aos fatores ambientais, associados à falta de higiene na manipulação e a um tempo mais prolongado de armazenamento, o medicamento esteja sujeito a sofrer alterações. 


\section{REFERÊNCIAS}

AGÊNCIA NACIONAL DE VIGILÂNCIA SANITÁRIA - ANVISA. Relatório: Painel internacional de avaliação da segurança da dipirona. Brasília, 2001. Disponível em: <http://www.anvisa.gov.br/divulga/informes/relatoriodipirona2.pdf>. Acesso em: 16 set. 2014.

BRASIL. Agência Nacional de Vigilância Sanitária (ANVISA). Resolução RE nº 1, de 29 de julho de 2005 - Determina sobre a estabilidade dos medicamentos. Disponível em: <http://www.anvisa.gov.br/medicamentos/legis/01_05_re>. Acesso em: 15 set. 2014.

BRASIL. Ministério da Saúde. Secretaria de Ciência, Tecnologia e Insumos Estratégicos. Departamento de Assistência Farmacêutica e Insumos Estratégicos. Diretrizes para estruturação de farmácias no âmbito do Sistema Único de Saúde. Brasília, 2009, p. 1-43. Disponível em: <http://www.sbrafh.org.br/site/public/tem/5162c 5812207f.pdf>. Acesso em: 15 set. 2014.

BUENO, C. S.; WEBER, D.; OLIVEIRA, K. R. Farmácia caseira e descarte de medicamentos no bairro Luiz Fogliatto do município de ljuí - RS. Revista de Ciências Farmacêuticas Básica e Aplicada, v. 30, n. 2, p.75-82, 2009.

CARVALHO J. P.; SANTOS, A. S.; SA, A. S.; TEIXEIRA, C. S.; NOGUEIRA, M. S. Estabilidade de medicamentos no âmbito da farmacovigilância. Revista Fármacos \& Medicamentos, vol. 9, n. 2, p. 22-27, jul./set. 2009.

FARMACOPEIA Brasileira, vol. 1. 5. ed. Agência Nacional de Vigilância Sanitária. Brasília: ANVISA, 2010a. p. 233-249.

FARMACOPEIA Brasileira, vol. 2. 5. ed. Agência Nacional de Vigilância Sanitária / Fundação Oswaldo Cruz. Brasília: ANVISA, 2010b. p. 912-914.

FERNANDES, L. C.; PETROVICK, P. R. Os medicamentos na farmácia caseira. In: SCHENKEL, E. P. Cuidados com os medicamentos. 4. ed. Porto Alegre: Editora da UFRGS, 2004. p. 39-42.

FERREIRA, W. A.; SILVA, M. E. S. T.; PAULA, A. C. C. F. F.; RESENDE, C. A. M. B. Avaliação de Farmácia Caseira no Município de Divinópolis (MG) por Estudantes do Curso de Farmácia da UNIFENAS. Revista Infarma, Brasília, v. 17, n. 7-9, p. 84-86, 2005. 
FERREIRA, M. B. C. Analgésicos Não Opioides. In: FUSCH, F. D.; WANNMACHER, L. Farmacologia Clínica: fundamentos da terapêutica racional. 4. ed. Rio de Janeiro: Guanabara Koogan, 2010. p. 342-378.

FERREIRA, B. C. A.; NOVAIS, E. B.; RIBEIRO, R. B. C.; FERNANDES, C. K. C. Estudo de estabilidade físico-química e microbiológica de dipirona em gotas armazenadas em residências do município de São Luis de Montes Belos-GO. Revista Faculdade Montes Belos, v. 7, n. 1, p. 109-120, 2014.

FIGUEIREDO, M. C.; SILVA, K. V. C. L.; BONACINA, C. M.; ORTIZ, F. T. Armazenagem de medicamentos em domicílios pelos moradores do bairro Figueirinha, em Xangri-lá, RS. Revista de Ciências Médicas e Biológicas, Salvador, v. 10, n. 2, p. 140-145, mai./ago. 2011.

GIL, E. S. Controle físico-químico de qualidade de medicamentos. 2. ed. São Paulo: Pharmabooks; 2007.

KNAPPMANN, A. L.; MELO, E. B. Qualidade de medicamentos isentos de prescrição: um estudo com marcas de dipirona comercializadas em uma drogaria de Cascavel. Revista Ciência \& Saúde Coletiva, Paraná, v. 15, n. 3, p. 3467-3476, 2010.

KÖHLER, L. F.; NASCIMENTO, H. D.; SCHWENGBER, E. L. L.; BANDEIRA, Z. M. P.; PAZIN, G. V.; MACHADO, S. R. P. Avaliação biofarmacotécnica e perfil de dissolução de comprimidos de dipirona: equivalências farmacêutica entre medicamentos de referência, genéricos e similares. Revista Brasileira de Farmácia, v. 90, n. 4, p. 309$315,2009$.

KOROLKOVAS, A.; BURCKHALTER, J. H. Química Farmacêutica. 1. ed. Rio de Janeiro: Editora Guanabara Koogan, 1988, p. 193-196.

MARGONATO F. B.; THOMSON Z.; PAOLIELLO M. M. B. Determinantes nas intoxicações medicamentosas agudas na zona urbana de um município do Sul do Brasil. Cadernos de Saúde Pública, v. 24, n. 2, p. 333-341, fev. 2008.

MARIN, N.; LUIZA, V. L.; CASTRO, C. G. S. O.; SANTOS, S. M. Assistência Farmacêutica para gerentes municipais. Rio de Janeiro: Organização Pan-Americana da Saúde / Organização Mundial da Saúde, 2003. Disponível em: <http://www.prefeitura.sp.gov.br/cidade/secretarias/upload/saude/arquivos/assistenci afarmaceutica/afgm.pdf>. Acesso em: 14 de out. de 2014. 
MASTROIANNI, P. C.; LUCCHETTA, R. C.; SARRA, J. R.; GALDURÓS, J. C. F. Estoque doméstico e uso de medicamentos em uma população cadastrada na estratégia saúde da família no Brasil. Revista Panamericana de Salud Pública, v. 29, n. 5, p. 358-364, 2011.

MILANEZ, M. C.; STUTZ, E.; ROSALES, T. O.; PENTEADO, A. J; PEREZ, E.;

CRUCIOL, J. M.; PEREIRA, E. M.; BOVO, F. Avaliação dos estoques domiciliares de medicamentos em uma cidade do Centro-Sul do Paraná. Revista de Ciências Médicas e Biológicas, Salvador, v. 12, n. 3, p. 283-289, set./dez. 2013.

NASCIMENTO, M. C. Medicamentos, comunicação e cultura. Revista Ciência \& Saúde Coletiva, Rio de Janeiro, v. 10, p. 179-193, set./dez. 2005.

PEIXOTO, M. M.; JÚNIOR, A. F. S.; SANTOS, C. A. A.; JÚNIOR, E. C. Avaliação da qualidade de comprimidos de captopril dispensados em Feira de Santana - BA. Revista Infarma, v. 16, n. 13-14, p. 69-73, 2005.

SANTOS, G.N.;ARAGÃO, C. C. V; FURLAN, C. M. Estudo da estabilidade acelerada de solução oral De paracetamol $200 \mathrm{mg} / \mathrm{ml}$, distribuída no Sistema Único de Saúde - SUS. Revista Brasileira de Ciências da Saúde, v. 7, n. 22, out./dez. 2009.

SERAFIM, E. O. P.; VECCHIO, A.; GOMES, J.; MIRANDA, A.; MORENO, A. H.; LOFFREDO, L. M. C.; SALGADO, H. R. N.; CHUNG, M. C. Qualidade dos medicamentos contendo dipirona encontrados nas residências de Araraquara e sua relação com a atenção farmacêutica. Revista Brasileira de Ciências Farmacêuticas, v. 43, n. 1, p. 127-135, jan./mar. 2007.

STULZER H. K.; SILVA M. A. Estudo de estabilidade de grânulos revestidos e comprimidos contendo Captopril. Acta Farmacêutica Bonaerense, v. 25, n. 4, p. 497$504,2006$.

TABORIANSKI A. M. Validação de métodos para análise e estudos de estabilidade de anti-retrovirais em preparações farmacêuticas. 2003. 167 f. Dissertação (Mestrado em Produção e Controle Farmacêuticos) - Programa de Pós-Graduação em Fármaco e Medicamentos, Faculdade de Ciências Farmacêuticas, Universidade de São Paulo, são Paulo, 2003. 
TOURINHO, F. S. V; BUCARETCHI, F.; STEPHAN, C.; CORDEIRO, R. Farmácias domiciliares e sua relação com a automedicação em crianças e adolescentes. Jornal de Pediatria - Scielo, Rio de Janeiro, v. 84, n. 5, p. 416-422, set./out. 2008.

TOTÓLI, E. G; STAIN, S. N.; SALGADO, H. R. N.; MORENO, A. H. Verificação da qualidade microbiológica de medicamentos contendo paracetamol encontrados em algumas residências de Américo Brasiliense/SP. Revista Uniara, v. 14, n. 2, dez. 2011.

WEST, D. S.; HERBERT, D. A.; KNOWLTON, C. H. A prática da Farmácia Comunitária. In: GENNARO, A. R. Remington: A Ciência e a Prática da Farmácia. 20. ed. vol. 1. Rio de Janeiro: Editora Guanabara Koogan, 2014. p. 30-34. 